\title{
THE DETERMINATION OF LOVE'S NUMBER $K$ FROM TIDAL VARIATIONS OF ROTATION OF A COMPRESSIBLE EARTH
}

\author{
N. N. PARIISKY and B. P. PERTSEV \\ Institute of Earth Physics, Moscow
}

\begin{abstract}
Long period bodily tides (fortnightly and monthly) cause periodic changes of the Earth's moment of inertia and consequently the angular speed of its rotation.

During the past few years the use of atomic clocks has made it possible to determine the amplitudes of these periodic variations of the Earth's rotation with high accuracy and has made this method very effective in determining Love's number $K$, which is dependent on the internal structure of the Earth (the most detailed studies were made in the U.S.S.R. - Pilnik and Gubanov).

But up to now it was taken (Anderson, Woolard, Melchior, and others), that the formulae of Jeffreys and Woolard, which are used in this method, are valid only for incompressible Earth models.

It is shown in this paper that the formulae are valid for the case of an actual compressible heterogeneous Earth. Thus, the method receives a real practical value. The use of lasers and radio methods in the near future will make this method even more effective.

The full text of the paper will be published in the magazine Physics of the Earth in No. 3, 1972.
\end{abstract}

\section{DISCUSSION}

H. Jeffreys: I think Professor Pariisky has been a little too generous to me. The relation of $k$ to the period of the free nutation, independent of compressibility, was used by Love and Larmor in the 19 th Century.

$N$. Pariisky: The question is to determine Love's number $k$ from amplitudes of fortnightly and monthly tidal variations of the Earth's rotation and not from the period of free nutation. The formulae for such determinations were first given by Sir Harold Jeffreys (Monthly Notices Roy. Astron. Soc. 2, 1928).

$P$. Melchior: It is very difficult to determine these long period zonal tides with geophysical instruments as the stability of such instruments is difficult to insure over periods such as 14 days. But these tides have maximum amplitude in polar regions. This was one of the objectives of the Spitsbergen astro-geo project. Prof. Bonatz succeeded in obtaining with his gravimeter a value of the Love factors combination of $\delta=1+h-\frac{3}{2} k=1.14$ for this zonal tide.

N. Pariisky: The results obtained by Prof. Bonatz are very interesting. Dr. Barsenkov, analysing a one year series of gravity observations at Talgar (Central Asia) with an Askania gravimeter has obtained $\delta=1.16$, the same value as Prof. Lecolazet obtained in Strasburg. But the determination of the Love number $k$ from tidal variations of the Earth's rotation has the advantage that it gives a more representative global value of $k$, and not the factor $\delta$, which is also a function of $h$. 